\title{
RESEARCH OF THE POWER PLANT OPERATIONAL MODES
}

\author{
Nina M. Koismynina ${ }^{1, *}$, and Timur T. Valiev ${ }^{1}$ \\ ${ }^{1}$ Tomsk Polytechnic University, 634050, Tomsk, Russia
}

\begin{abstract}
In this article the algorithm of the power plant operational modes research is offered. According to this algorithm the program for the modes analysis and connection power transformers choice is developed. The program can be used as educational means for studying of the power plant electric part, at the same time basic data are provided. Also the program can be used for the analysis of the working power plants modes. Checks of the entered data completeness and a choice correctness of the operational modes are provided in the program; in all cases of a deviation from the correct decisions to the user the relevant information is given.
\end{abstract}

\section{Introduction}

The most important problems of power supply system functioning and management are the equipment structure choice and the power plants modes analysis [1]. The electric power isdeveloped at power plants of a different type depending on technological process: thermal, nuclear, hydraulic. According to the Russian Federation Energy Ministry in 2016 year $65.41 \%$ of the developed power were the corresponds to the thermal power plants, $15.4 \%$ - to the hydraulic, $19.3 \%$ - to the nuclear power plants. Thermal and nuclear power plants turbogenerators with the drive from steam turbines are established. Powerful thermal and nuclear power plants as the installed turbines are condensation.

Feature of condensation power plants block diagrams is power development from generators to power transformers and to voltage switchgear [2]. As a rule, condensation power plants have two voltage switchgear: high voltage switchgear (HV switchgear) and the middle voltage switchgear (MV switchgear). The electric power on HV switchgear power lines is transferred to substations HV switchgear. Substations electric power will be transformed to the required level of distributing devices voltage - low voltage switchgear (LV switchgear). The powerful substations can have the middle voltage switchgear (MV switchgear) [2]. The example of the condensation power plants block diagram with three winding connection transformers is presented in Fig.1. The power plant has three turbogenerators $\left(\mathrm{G}_{1}, \mathrm{G}_{2}, \mathrm{G}_{3}\right)$. The $\mathrm{G}_{1}$ generator is connected to a lowest voltage winding of the $T_{1}$ transformer which works at $\mathrm{HV}$ switchgear. The $\mathrm{G}_{2}$ generator is connected to a lowest voltage winding of the $T_{2}$ transformer which works at MV switchgear. The $\mathrm{G}_{3}$ generator is connected to a lowest voltage winding of the $T_{3}$ transformer which works at MV switchgear. Communication between HV switchgear and MV switchgear is carried out

* Corresponding author: kosm_nm@tpu.ru 
by three winding transformers $\mathrm{T}_{4}$ and $\mathrm{T}_{5}$. At certain combinations of voltage instead of the three winding transformers autotransformers are used; in this article this case isn't considered. The HV switchgear has connection with a power supply system (in drawing it is designated - a source S) and external consumers loading by the total power of $\mathrm{S}_{\mathrm{HV}}$. The MV switchgear has middle voltage external consumers loading of the total power of $S_{M V}$. Power supply of power plant internal consumers (own needs) is carried out from blocks the generator - transformer and is designated as c.n; reserve food of internal consumers from the connection transformers is also shown.

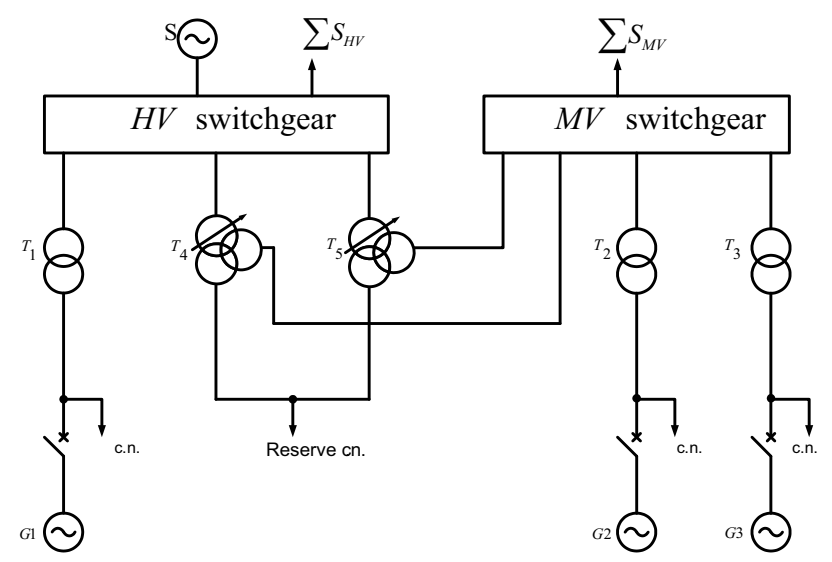

Fig. 1. The power plant with the connection transformer block diagram.

\section{Experimental setup and study technique}

At power plant the following operational modes are possible.

Load modes: the maximum load of MV switchgear, the minimum load mode of MV switchgear, the maximum load mode of HV switchgear, the minimum load HV switchgear. Repair mode: removal from work of one connection transformers. Postemergency mode: shutdown of one block generator - the transformer from switchgear (HV or MV) leads to emergence of postemergency mode as this shutdown is possible only at block equipment accident and it removal from work. Each of the described modes (or their combinations) is characterized by the balances capacities of power plant characteristic point: turbogenerators stator winding, switchgear (Fig. 2), neutral points of three winding transformers equivalent circuits.

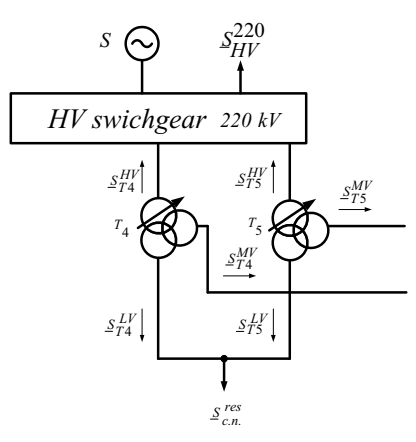

Fig. 2. HV switchgear point.

For research only those operational modes at which there is a change of connection 
transformers overflows are chosen. The required rated power the three winding power transformers is made after definition the capacities balances equations and calculation of power overflows: $\mathrm{S}_{\mathrm{RATE}}=\max \left(\mathrm{S}_{\mathrm{LW}}, \mathrm{S}_{\mathrm{MV}}, \mathrm{S}_{\mathrm{HV}}\right)$, where $\mathrm{S}_{\mathrm{LW}}-$ power overflow on a low voltage winding; $\quad \mathrm{S}_{\mathrm{MV}}$ - power overflow on a middle voltage winding; $\mathrm{S}_{\mathrm{HV}}$ - power overflow on a high voltage winding.

In practice of the power plant modes analysis it is accepted not to consider losses of power transformers. In this case for condensation power plant with connection transformers above the given formula becomes simpler as $\mathrm{S}_{\mathrm{MV}}=\mathrm{S}_{\mathrm{HV}}-$ at not accounting of power transformers losses and here is also a working food of internal consumers. Therefore, $\mathrm{S}_{\mathrm{RATE}}$ $=\mathrm{S}_{\mathrm{LW}}$.

Similar calculations are carried out for each operational mode, and the largest required power for the connection transformer is defined.

\section{Experimental results}

The program for research of power plant operational modes is carried on Electric Power Systems Department of National Research Tomsk Polytechnic University Power Engineering Institute. This development is further work on re-equipment of the department laboratory modern software [3].

The program is written in the Delphi programming language which has a number of advantage in comparison with similar products: speed of development; low requirements to resources to computers; building due to embedding new a component and tools; possibility of development of new components and tools own means; successful study of hierarchy of objects [4].

The program consists of the next windows: the description, the basic data input, the block diagram formation, the operational mode choice, the calculation results (the program work protocol with his subsequent preservation in the file form). The description window contains the program name and assignment, the executed functions, data on the developer and the research supervisor. The input generator parameters window contains the fields: rated active power, stator winding voltage, power factor, electric power expense on power plant own needs, number of generators (Fig. 3). The data input window on loadings contains the fields: maximum active power of one loading line, power factor, coefficient of simultaneity of loading maxima, number of lines, voltage; for HV loading only voltage is requested.

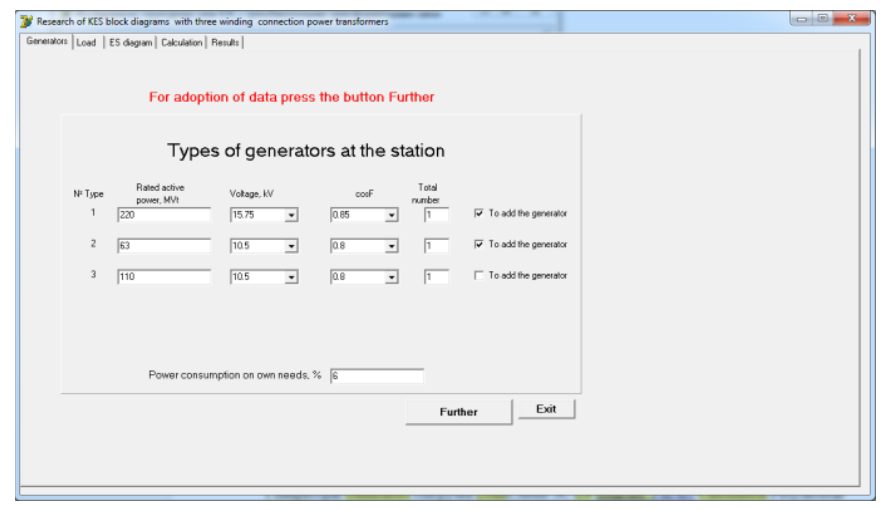

Fig. 3. Input window of power plant generators parameters. 
For formation of the power plant block diagram the user needs to execute input of connection transformers number (from 1 to 3 ) and the number of generator-transformer blocks connected to MV switchgear and HV switchgear (Fig.4).

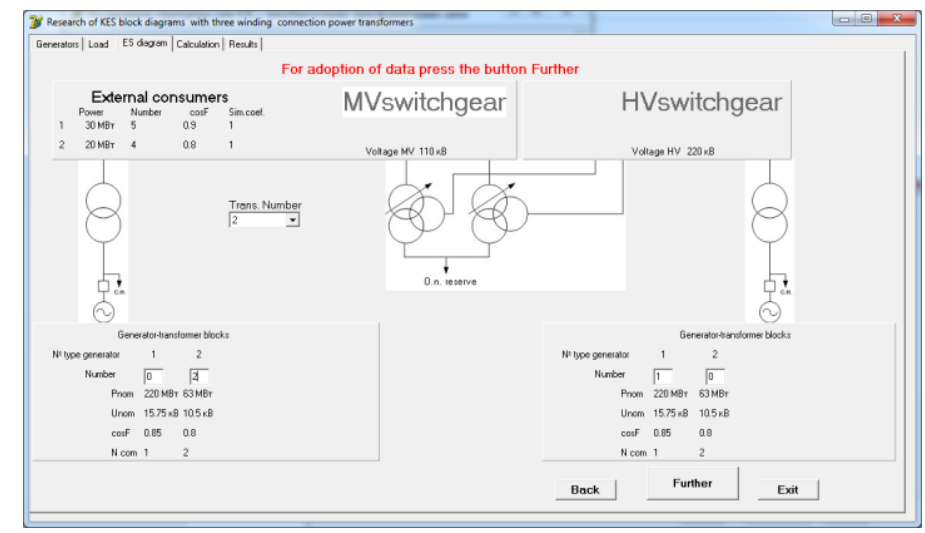

Fig. 4. Window of the power plant block diagram formation.

The window of the station operational mode choice is described below. In this window the user chooses station mode, and for postemergency operation mode obligatory specifies type of the switched-off generator and a power expense on own needs for maintenance of a disconnected equipment normal state.

For the aid to the user checks in equipment nominal parameters and also power plant structure and the studied operational modes are provided in the program. So, generators rated power, own needs (internal consumers) power expense coefficients; power factors for external consumers; simultaneity coefficients of loadings maxima for external consumers; value of switchgear voltage; the block generators number, used in the block diagram; feasibility of the operational mode set users for the concrete block diagram are checked.

\section{Conclusion}

The presented material contains the concentrated statement of operational power plant mode and calculation algorithm of power connection power transformers overflows

On this basis the program allowing according to the entered data is developed: to create the power plant block diagram; to set the operational mode; to calculate connection transformers power overflows; to create the calculation protocol; to keep calculation data in the file; to study reference methodical material on this subject.

Now work on introduction of the developed program for educational and research tasks performance in TPU is conducted.

\section{References}

1. Y. Gusev, Thermal Engineering, 3, 62 (2015)

2. B. Starshinov, M. Piratorov, M. Kozinova, Electric part of power plants and substations (MEI, Moscow, 2015) [in Russian]

3. I. Tsoy, N. Kosmynina, IOP Conference Series: Earth and Environmental Science, 6, 27 (2015)

4. A. Arkhangelsk, Programming in Delphi: the textbook according to classical versions (Binom, Moscow, 2008) [in Russian] 